\title{
Existence, Asymptotic Behaviour, and Blow up of Solutions for a Class of Nonlinear Wave Equations with Dissipative and Dispersive Terms
}

\author{
Necat Polat ${ }^{\mathrm{a}}$ and Doğan Kaya ${ }^{\mathrm{b}}$ \\ a Dicle University, Department of Mathematics, 21280 Diyarbakir, Turkey \\ b Ardahan University, Engineering Faculty, 75100 Ardahan, Turkey \\ Reprint requests to N. P.; E-mail: npolat@ dicle.edu.tr
}

Z. Naturforsch. 64a, 315 - 326 (2009); received April 22, 2008 / revised August 11, 2008

\begin{abstract}
We consider the existence, both locally and globally in time, the asymptotic behaviour, and the blow up of solutions to the initial boundary value problem for a class of nonlinear wave equations with dissipative and dispersive terms. Under rather mild conditions on the nonlinear term and the initial data we prove that the above-mentioned problem admits a unique local solution, which can be continued to a global solution, and the solution decays exponentially to zero as $t \rightarrow+\infty$. Finally, under a suitable condition on the nonlinear term, we prove that the local solutions with negative and nonnegative initial energy blow up in finite time.
\end{abstract}

Key words: Nonlinear Wave Equation; Initial Boundary Value Problem; Global Solution; Asymptotic Behaviour; Blow up of Solutions.

\section{Introduction}

We are concerned with the existence, both locally and globally in time, the asymptotic behaviour, and the blow up of solutions to the initial boundary value problem for the following class of nonlinear wave equations with dissipative and dispersive terms:

$$
\begin{aligned}
& u_{t t}-u_{x x}-u_{x x t t}-\lambda u_{x x t}+u=\sigma\left(u_{x}\right)_{x}, \\
& (x, t) \in(0,1) \times(0,+\infty), \\
& u(0, t)=u(1, t)=0, \quad t \geq 0, \\
& u(x, 0)=u_{0}(x), \quad u_{t}\left(x_{0}\right)=u_{1}(x), \quad x \in[0,1],
\end{aligned}
$$

where $\lambda$ is a real number and $\sigma(s)$ is a given nonlinear function.

Physically, in real processes, the dissipation and dispersion have an important role for the energy amplification arising from the nonlinearity, and their interaction with the nonlinearity accompanies the accumulation, balance, and dissipation of the energy, see [1]. Many mathematicians and physicists focus their attention to study nonlinear evolution equations with dissipative or dispersive terms or with both of them. There are a lot of references investigating in detail the restriction conditions among the nonlinearity, the dispersion, and the dissipation, see [1-34].
The well known viscoelastic equation

$$
u_{t t}-u_{x x t}=\sigma\left(u_{x}\right)_{x}
$$

is an important class of nonlinear evolution equations which was suggested from the longitudinal displacement in a homogeneous rod with nonlinear strain and viscosity [5]. The dissipative term $u_{x x t}$, arising from the viscoelastic bar material, makes the initial boundary value problem of (4) more tractable than that of the one-dimensional nonlinear elasticity

$$
u_{t t}=\sigma\left(u_{x}\right)_{x} .
$$

There are many results $[2,6,9,10,32]$ on the global existence, nonexistence and blow up, smoothness and asymptotic behaviours of solutions for the initial boundary value problem of (4).

In [32], Zhijian and Changming studied the blow up of solutions for the initial boundary value problem of (4).

In $[35,36]$, another class of nonlinear wave equations

$$
u_{t t}-u_{x x}-u_{x x t t}=a\left(u_{s}^{n}\right)_{x}
$$

was suggested in studying the transmission of nonlinear waves in a nonlinear elastic rod.

In [37], Guowang and Shubin proved the existence and uniqueness of a classical global solution and the 
blow up of solutions to the initial boundary value problem for the equation

$$
u_{t t}-\alpha u_{x x}-\beta u_{x x t t}=\varphi\left(u_{x}\right)_{x} .
$$

Finally they applied the results of the above problem to the equation arising for nonlinear waves in elastic rods:

$$
u_{t t}-\left[a_{0}+n a_{1}\left(u_{x}\right)^{n-1}\right] u_{x x}-a_{2} u_{x x t t}=0 .
$$

Yacheng and Junsheng [31] studied the global existence, the asymptotic behaviour, and the blow up of $W^{k, p}$ solutions to the initial boundary value problem for the equation

$$
u_{t t}-\alpha u_{x x t}-u_{x x t t}=\sigma\left(u_{x}\right)_{x} .
$$

Zhijian [33] studied the global existence, asymptotic behaviour, and blow up of solutions to the initial boundary value problem for a class of nonlinear wave equations with a dissipative term:

$$
u_{t t}+\Delta^{2} u+\lambda u_{t}=\sum_{i=1}^{N} \frac{\partial}{\partial x_{i}} \sigma_{i}\left(u_{x_{i}}\right) .
$$

Polat et al. [23] established the blow up of solutions of the initial boundary value problem for a class of nonlinear wave equations with a damping term:

$$
u_{t t}=\operatorname{div} \sigma(\nabla u)+\Delta u_{t}-\Delta^{2} u .
$$

Zhijian [34] has studied the existence, both locally and globally in time, the decay estimates, and the blow up of solutions to the Cauchy problem for a class of nonlinear dispersive wave equations arising in elastoplastic flow:

$$
u_{t t}+u_{x x x x}+\lambda u=\sigma\left(u_{x}\right)_{x}
$$

and investigated the influence of the dispersive term $\lambda u$ for the corresponding solutions.

Levandosky [15] studied the local existence and decay estimates of solutions to the Cauchy problem of the equation

$$
u_{t t}+\Delta^{2} u+u=f(u)
$$

Throughout the present paper, we use the following abbreviations and lemmas:

$$
L^{p}=L^{p}[0,1] \quad\|\cdot\|_{L^{p}}=\|\cdot\|_{p},
$$

$$
\|\cdot\|_{W^{k, p}[0,1]}=\|\cdot\|_{k, p}, \quad(u, v)=\int_{0}^{1} u v \mathrm{~d} x .
$$

In order to simplify the exposition, different positive constants might be denoted by the same letter $C$.

Lemma $1[31,33,38]$. Let $\Omega \in \mathrm{R}^{n}$ be a bounded domain $k \geq 0,1 \leq p \leq \infty$. Assume that $G\left(z_{1}, \ldots, z_{h}\right) \in C^{k}\left(\mathrm{R}^{h}\right), z_{i}(x, t) \in L^{\infty}\left([0, T] ; W^{k, p}(\Omega)\right)$ $(i=1, \ldots, h) \quad$ and $\left\|z_{i}\right\|_{L^{\infty}\left([0, T] ; L^{\infty}(\Omega)\right)}$. Then $G\left(z_{1}, \ldots, z_{h}\right) \in L^{\infty}\left([0, T] ; W^{p, k}(\Omega)\right)$ and

$$
\begin{array}{r}
\left\|G\left(z_{1}, \ldots, z_{h}\right)\right\|_{L^{\infty}\left([0, T] ; W^{p, k}(\Omega)\right)} \leq \\
C(M) \sum_{i=1}^{h}\left\|z_{i}\right\|_{L^{\infty}\left([0, T] ; W^{p, k}(\Omega)\right)},
\end{array}
$$

where

$$
\|z\|_{L^{\infty}\left([0, T] ; W^{p, k}(\Omega)\right)}=e s s \sup _{0 \leq t \leq T}\|z(t)\|_{k, p} .
$$

Lemma 2 [31]. Assume that $f(s) \in C^{m+1}(\mathrm{R}), u, v \in$ $L^{\infty}\left([0, T] ; W^{k, p}[0,1]\right), m \geq 1$, and $1<p<\infty$. Then

$$
\begin{aligned}
& \|f(u)-f(v)\|_{m, p} \leq C\left(\|u\|_{m, p},\|v\|_{m, p}\right)\|u-v\|_{m, p}, \\
& 0 \leq t \leq T .
\end{aligned}
$$

Lemma $3[31,39,40]$. Let $\Omega \in \mathrm{R}^{n}$ be a bounded domain, and $u(x) \in W^{1,2}(\Omega)$ be the unique solution of problem

$$
\begin{aligned}
& u-\Delta u=f(x), \quad x \in \Omega, \\
& \left.u\right|_{\partial \Omega}=0 .
\end{aligned}
$$

Assume that $f(x) \in W^{k, p}(\Omega), k \geq 0$, and $1<p<\infty$, then $u(x) \in W^{k+2, p}(\Omega)$ and

$$
\|u\|_{k+2, p} \leq C\|f\|_{k, p} .
$$

Remark 1. Take $u=u(x, t)$ and $f=f(x, t)$ in Lemma 3, then the result of the lemma implies that $(I-$ $\Delta)^{-1}: L^{\infty}\left([0, T] ; W^{k, p}(\Omega)\right) \rightarrow L^{\infty}\left([0, T) ; W^{k+2, p}(\Omega) \cap\right.$ $\left.W_{0}^{1, p}(\Omega)\right)$ and

$$
\left\|(I-\Delta)^{-1} f\right\|_{L^{\infty}\left([0, T], W^{k+2, p}(\Omega)\right)} \leq C\|f\|_{L^{\infty}\left([0, T], W^{k, p}(\Omega)\right)},
$$

where $(I-\Delta)^{-1} f=\int_{\Omega} K(x, y) f(y) \mathrm{d} y$, and $K(x, y)$ is the Green function of problem (5).

Lemma 4 [31,41-43]. Let $K(x, y)$ be the Green function of the boundary value problem for the ordinary differential equation

$$
y(x)-y^{\prime \prime}(x)=0, \quad y(0)=y(1)=0,
$$


i.e.,

$$
K(x, \xi)=\frac{1}{\sinh 1} \begin{cases}\sinh (1-\xi) \sinh x, & 0 \leq x<\xi, \\ \sinh \xi \sinh (1-x), & \xi \leq x \leq 1 .\end{cases}
$$

The Green function $K(x, \xi)$ satisfies the following properties:

(1) $K(x, \xi)$ is defined and continuous in $Q=\{0 \leq$ $x \leq 1,0 \leq \xi \leq 1\}$.

(2) $K(x, \xi)$ satisfies the homogeneous equation

$$
K(x, \xi)-K_{x x}(x, \xi)=0, \quad x \neq \xi
$$

and the homogeneous conditions

$$
K(0, \xi)=0, \quad K(1, \xi)=0 .
$$

(3) $K_{x}(x, \xi)$ has a point of discontinuity of the first kind at $x=\xi$ and satisfies the condition

$$
K_{x}(\xi+0, \xi)-K_{x}(\xi-0, \xi)=-1 .
$$

(4) $K(x, \xi)=K(\xi, x)$.

(5) $0 \leq K(x, \xi)<\frac{2}{7}, \quad 0 \leq x \leq 1, \quad 0 \leq \xi \leq 1$.

(6) $\left|K_{\xi, x}(x, \xi)\right| \leq C, \quad x \neq \xi$.

The paper is organized as follows. First of all, we reduce problem (1) - (3) to an equivalent integral equation by means of the Green function of a boundary value problem for the second-order ordinary differential equation (6). Then making use of the contraction mapping principle we prove the existence and uniqueness of the local solutions for the integral equation in Section 2. Under some conditions by use of a priori estimates of the solution we prove in Section 3 that problem (1) - (3) has a unique global solution. The proof of the asymptotic behaviour of the global solutions is given in Section 4. In Section 5, the blow up of solutions for problem (1) - (3) is given.

\section{Existence and Uniqueness of Local Solutions}

In this section we prove the existence and the uniqueness of the local solutions for problem (1)-(3) by the contraction mapping principle.

For this purpose let $K(x, \xi)$ be the Green function of problem (6); we can rewrite (1) as follows:

$$
\left[u_{t t}+u\right]-\left[u_{t t}+u\right]_{x x}=\sigma\left(u_{x}\right)_{x}+\lambda u_{x x t} .
$$

From (7) and the solution of (1) satisfying condition (2), we get

$$
\begin{aligned}
& u_{t t}(x, t)+u(x, t)= \\
& \quad\left(I-\frac{\partial^{2}}{\partial x^{2}}\right)^{-1}\left(\sigma\left(u_{x}(x, t)\right)_{x}+\lambda u_{x x t}(x, t)\right) \\
& \equiv \int_{0}^{1} K(x, \xi)\left[\sigma\left(u_{\xi}(\xi, t)\right)_{\xi}+\lambda u_{\xi \xi t}(\xi, t)\right] \mathrm{d} \xi
\end{aligned}
$$

From (3) and (8) we know that the initial boundary value problem (1)-(3) is equivalent to the integral equation

$$
\begin{aligned}
u(x, t)= & u_{0}(x)+u_{1}(x) t-\int_{0}^{t}(t-\tau) u(x, \tau) \mathrm{d} \tau \\
& +\int_{0}^{t}(t-\tau)\left(I-\frac{\partial^{2}}{\partial x^{2}}\right)^{-1}\left(\sigma\left(u_{x}(x, \tau)\right)_{x}\right. \\
& \left.+\lambda u_{x x \tau}(x, \tau)\right) \mathrm{d} \tau \\
\equiv & u_{0}(x)+u_{1}(x) t-\int_{0}^{t}(t-\tau) u(x, \tau) \mathrm{d} \tau \\
& +\int_{0}^{t} \int_{0}^{1}(t-\tau) K(x, \xi)\left[\sigma\left(u_{\xi}(\xi, \tau)\right)_{\xi}\right. \\
& \left.+\lambda u_{\xi \xi \tau}(\xi, \tau)\right] \mathrm{d} \xi \mathrm{d} \tau .
\end{aligned}
$$

Now we are going to prove the existence and the uniqueness of the local solution for the integral equation (9) by the contraction mapping principle.

Let us define the function space

$$
\begin{array}{r}
X_{k}(T)=\left\{u(x, t) \in W^{1, \infty}\left([0, T] ; W^{k, p}[0,1] \cap W_{0}^{1, p}[0,1]\right),\right. \\
u(0, t)=u(1, t)=0\},
\end{array}
$$

which is endowed with the norm

$$
\begin{aligned}
& \|u\|_{X_{k}(T)}=\|u\|_{W^{1, \infty}\left([0, T] ; W^{k, p}[0,1]\right)} \\
& =\|u\|_{L^{\infty}\left([0, T] ; W^{k, p}[0,1]\right)}+\left\|u_{t}\right\|_{L^{\infty}\left([0, T] ; W^{k, p}[0,1]\right)}, \\
& \forall u \in X_{k}(T) .
\end{aligned}
$$

It is easy to see that $X_{k}(T)$ is a Banach space. Let $M=$ $\left\|u_{0}\right\|_{k, p}+\left\|u_{1}\right\|_{k, p}$. Take the set

$$
\begin{gathered}
Y_{k}(M, T)=\left\{u \mid u \in W^{1, \infty}\left([0, T] ; W^{k, p}[0,1] \cap W_{0}^{1, p}[0,1]\right),\right. \\
\left.\|u\|_{X_{k}(T)} \leq M+2\right\} .
\end{gathered}
$$

Obviously, $Y_{k}(M, T)$ is a nonempty, bounded, closed convex subset of $X_{k}(T)$ for any fixed $M>0$ and $T>0$. 
Define the map $H$ as

$$
\begin{gathered}
H u(x, t)=u_{0}(x)+u_{1}(x) t-\int_{0}^{t}(t-\tau) u(x, \tau) \mathrm{d} \tau \\
+\int_{0}^{t}(t-\tau)\left(I-\frac{\partial^{2}}{\partial x^{2}}\right)^{-1} \\
\cdot\left(\sigma\left(u_{x}(x, \tau)\right)_{x}+\lambda u_{x x \tau}(x, \tau)\right) \mathrm{d} \tau
\end{gathered}
$$

where $u \in X_{k}(T)$. We can easily show that $H$ maps $X_{k}(T)$ into $X_{k}(T)$. If $\sigma(s) \in C^{k-1}(\mathrm{R}), k \geq 2,1<p<\infty$, then from (10) and Lemma 1 we have

$$
\begin{aligned}
& u_{x} \in W^{1, \infty}\left([0, T] ; W^{k-1, p}[0,1] \cap L^{\infty}[0,1]\right), \\
& \sigma\left(u_{x}\right) \in L^{\infty}\left([0, T] ; W^{k-1, p}[0,1]\right)
\end{aligned}
$$

and $\sigma\left(u_{x}\right)_{x}+\lambda u_{x x t} \in L^{\infty}\left([0, T] ; W^{k-2}[0,1]\right)$. By Lemma 3 we get

$$
\begin{aligned}
& \left(I-\frac{\partial^{2}}{\partial x^{2}}\right)^{-1}\left(\sigma\left(u_{x}\right)_{x}+\lambda u_{x x t}\right) \\
& \quad \in L^{\infty}\left([0, T] ; W^{k, p}[0,1] \cap W_{0}^{1, p}[0,1]\right)
\end{aligned}
$$

and $H u \in X_{k}(T)$.

Our goal is to show that $H$ has a unique fixed point in $Y_{k}(M, T)$ for suitable $T$.

Theorem 1. Assume that $u_{0}, u_{1} \in W^{k, p}[0,1] \cap$ $W_{0}^{1, p}[0,1]$ and $\sigma(s) \in C^{k}(\mathrm{R}), k \geq 2,1<p<\infty$. Then $H$ is a contractive mapping from $Y_{k}(M, T)$ into itself for $T$ sufficiently small relative to $M$. Then problem (1)-(3) admits a unique solution $u(x, t) \in$ $W^{2, \infty}\left(\left[0, T_{0}\right) ; W^{k, p}[0,1] \cap W_{0}^{1, p}[0,1]\right)$, where $\left[0, T_{0}\right)$ is the maximal time interval of existence for $u(x, t)$.

Proof. We first prove that $H$ maps $Y_{k}(M, T)$ into itself for $T$ small enough. Let $u \in Y_{k}(M, T)$ be given. From (11) we get

$$
\begin{aligned}
& \|H u\|_{k, p} \leq\left\|u_{0}\right\|_{k, p}+\left\|u_{1}\right\|_{k, p} T \\
& +\int_{0}^{t}(t-\tau)\|u\|_{k, p} \mathrm{~d} \tau \\
& +\int_{0}^{t}(t-\tau)\left\|\left(I-\frac{\partial^{2}}{\partial x^{2}}\right)^{-1}\left(\sigma\left(u_{x}\right)_{x}+\lambda u_{x x \tau}\right)\right\|_{k, p} \mathrm{~d} \tau .
\end{aligned}
$$

Using Lemma 3 and Lemma 1, it follows easily that

$$
\begin{aligned}
& \|u\|_{k, p} \leq M+2, \\
& \left\|\left(I-\frac{\partial^{2}}{\partial x^{2}}\right)^{-1}\left(\sigma\left(u_{x}\right)_{x}+\lambda u_{x x t}\right)\right\|_{k, p} \\
& \leq C\left\|\sigma\left(u_{x}\right)_{x}+\lambda u_{x x t}\right\|_{k-2, p} \leq C(M)(M+2) .
\end{aligned}
$$

Substituting inequality (14) into (13) we obtain

$$
\begin{aligned}
\|H u\|_{k, p} \leq & \left\|u_{0}\right\|_{k, p}+\left\|u_{1}\right\|_{k, p} T \\
& +\frac{1}{2}(C(M)+1)(M+2) T^{2} .
\end{aligned}
$$

On the other hand, from (11) and (14) we get

$$
\begin{aligned}
&(H u)_{t}= u_{1}-\int_{0}^{t} u \mathrm{~d} \tau \\
&+\int_{0}^{t}\left(I-\frac{\partial^{2}}{\partial x^{2}}\right)^{-1}\left(\sigma\left(u_{x}\right)_{x}+\lambda u_{x x \tau}\right) \mathrm{d} \tau \\
&\left\|(H u)_{t}\right\|_{k, p} \leq\left\|u_{1}\right\|_{k, p}+(C(M)+1)(M+2) T
\end{aligned}
$$

Thus from (15) and (16) we have

$$
\begin{aligned}
\|H u\|_{X_{k}(T) \leq} & M+(M+(C(M)+1)(M+2)) T \\
& +\frac{1}{2}(C(M)+1)(M+2) T^{2} .
\end{aligned}
$$

If $T$ satisfies

$$
\begin{aligned}
t \leq \min \left\{\frac{1}{M+(C(M)+1)(M+2)},\right. \\
{\left.\left[\frac{2}{(C(M)+1)(M+2)}\right]^{1 / 2}\right\}, }
\end{aligned}
$$

then

$$
\|H u\|_{X_{k}(T)} \leq M+2 .
$$

Therefore, if condition (17) holds, then $H$ maps $Y_{k}(M, T)$ into $Y_{k}(M, T)$.

Let $T>0$ and $u, v \in Y_{k}(M, T)$ be given. We have

$$
\begin{aligned}
\|H u-H v\|_{k, p} \leq & \int_{0}^{t}(t-\tau)\|u-v\|_{k, p} \mathrm{~d} \tau \\
+\int_{0}^{t}(t-\tau) \|\left(I-\frac{\partial^{2}}{\partial x^{2}}\right) & \|\left(\sigma\left(u_{x}\right)-\sigma\left(v_{x}\right)\right)_{x} \\
& \left.+\lambda(u-v)_{x x \tau}\right] \|_{k, p} \mathrm{~d} \tau .
\end{aligned}
$$


By Lemma 3 and Lemma 2, we obtain

$$
\begin{gathered}
\left\|\left(I-\frac{\partial^{2}}{\partial x^{2}}\right)^{-1}\left[\left(\sigma\left(u_{x}\right)-\sigma\left(v_{x}\right)\right)_{x}+\lambda(u-v)_{x x t}\right]\right\|_{k, p} \\
\leq C\left[C(M)\|u-v\|_{k, p}+\left\|(u-v)_{t}\right\|_{k, p}\right] .
\end{gathered}
$$

Substituting inequality (20) into (19) we obtain

$$
\|H u-H v\|_{k, p} \leq \frac{1}{2} C(M) T^{2}\|u-v\|_{X_{k}(T)} .
$$

On the other hand, from (11) and (20) we get

$$
\left\|(H u-H v)_{t}\right\|_{k, p} \leq C(M) T\|u-v\|_{X_{k}(T)} .
$$

Thus from (21) and (22) we have

$$
\begin{aligned}
\|H u-H v\|_{X_{k}(T)} & \leq C(M) T\|u-v\|_{X_{k}(T)} \\
& +\frac{1}{2} C(M) T^{2}\|u-v\|_{X_{k}(T)} .
\end{aligned}
$$

Take $T$ satisfying (17) and

$$
T<\min \left\{\frac{1}{2 C(M)},\left[\frac{1}{C(M)}\right]^{1 / 2}\right\} .
$$

Then

$$
\|H u-H v\|_{X_{k}(T)}<\|u-v\|_{X_{k}(T)} .
$$

This shows that $H: Y_{k}(M, T) \rightarrow Y_{k}(M, T)$ is strictly contractive.

From (18) and (24) and the contraction mapping principle, for appropriately chosen $T>0, H$ has a unique fixed point $u(x, t) \in Y_{k}(M, T)$, which is a unique solution of problem (1) - (3). And from (8) and (12) we have

$$
\begin{gathered}
u_{t t}(x, t)+u(x, t)=\left(I-\frac{\partial^{2}}{\partial x^{2}}\right)^{-1}\left(\sigma\left(u_{x}\right)_{x}+\lambda u_{x x t}\right) \\
\in L^{\infty}\left([0, T] ; W^{k, p}[0,1] \cap W_{0}^{1, p}[0,1]\right) .
\end{gathered}
$$

Thus (25) implies

$$
u(x, t) \in W^{2, \infty}\left(\left[0, T_{0}\right) ; W^{k, p}[0,1] \cap W_{0}^{1, p}[0,1]\right),
$$

where $\left[0, T_{0}\right)$ is the maximal time interval of existence for $u \in X_{k}\left(T_{0}\right)$. This completes the proof of the theorem.

Now, we discuss the global existence and uniqueness of solutions.

\section{Existence and Uniqueness of Global Solutions}

In this section, we prove the existence and uniqueness of the global solutions for problem (1)-(3). For this purpose we are going to make a priori estimates of the local solutions for problem (1) - (3) and we suppose that the conditions of Theorem 1 hold.

Theorem 2. Assume that $u_{0}, u_{1} \in W^{2, p}[0,1] \cap$ $W_{0}^{1, p}[0,1], \sigma(s) \in C^{2}(\mathrm{R}), 1<p<\infty$, and the following conditions hold:

(i) $\sigma(s) s \geq 0, s \in \mathrm{R}$;

(ii) $|\sigma(s)| \leq C_{1} \int_{0}^{s} \sigma(y) \mathrm{d} y+C_{2}, s \in \mathrm{R}$, where $C_{1}$ and $C_{2}$ are positive constants.

Then for any $T>0$ problem $(1)-(3)$ admits a unique global solution $u(x, t) \in W^{2, \infty}([0, T]$; $\left.W^{2, p}[0,1] \cap W_{0}^{1, p}[0,1]\right)$.

Proof. Taking the $L^{2}$ inner product with $u_{t}$ in (1) and integrating the resulting expression over $[0, t]$ we get

$$
\begin{gathered}
\|u(t)\|_{H^{1}}^{2}+\left\|u_{t}(t)\right\|_{H^{1}}^{2}+2 \int_{0}^{1} F\left(u_{x}\right) \mathrm{d} x \\
+2 \lambda \int_{0}^{t}\left\|u_{x \tau}(\tau)\right\|_{2}^{2} \mathrm{~d} \tau= \\
\left\|u_{0}\right\|_{H^{1}}^{2}+\left\|u_{1}\right\|_{H^{1}}^{2}+2 \int_{0}^{1} F\left(u_{0 x}\right) \mathrm{d} x
\end{gathered}
$$

where $F(s)=\int_{0}^{s} \sigma(y) \mathrm{d} y$.

If $\sigma(s) s \geq 0, s \in \mathrm{R}$, then $F(s) \geq 0$. Thus from (27) we have

$$
\begin{aligned}
& \|u(t)\|_{H^{1}}^{2}+\left\|u_{t}(t)\right\|_{H^{1}}^{2}+2 \int_{0}^{1} F\left(u_{x}\right) \mathrm{d} x \leq\left\|u_{0}\right\|_{H^{1}}^{2} \\
& +\left\|u_{1}\right\|_{H^{1}}^{2}+2 \int_{0}^{1} F\left(u_{0 x}\right) \mathrm{d} x+2|\lambda| \int_{0}^{t}\left\|u_{x \tau}(\tau)\right\|_{2}^{2} \mathrm{~d} \tau .
\end{aligned}
$$

From the above inequality and the Gronwall inequality we get

$$
\|u(t)\|_{H^{1}}^{2}+\left\|u_{t}(t)\right\|_{H^{1}}^{2}+2 \int_{0}^{1} F\left(u_{x}\right) \mathrm{d} x \leq C(T)
$$

and

$$
\|u(t)\|_{H^{1}}^{2}+\left\|u_{t}(t)\right\|_{H^{1}}^{2} \leq C(T), \quad 0 \leq t \leq T
$$

where $C(t)$ is a constant dependent on $T$. 
With partial integration of (28), we obtain

$$
\begin{aligned}
& u_{t t}(x, t)+u(x, t) \\
& =\int_{0}^{1} K(x, \xi)\left[\sigma\left(u_{\xi}(\xi, t)\right)_{\xi}+\lambda u_{\xi \xi t}(\xi, t)\right] \mathrm{d} \xi \\
& =-\int_{0}^{1} K_{x}(x, \xi)\left[\sigma(u \xi(\xi, t))+\lambda u_{\xi t}(\xi, t)\right] \mathrm{d} \xi .
\end{aligned}
$$

Differentiating the above equation with respect to $x$ and using (3) of Lemma 4 it follows that

$$
\begin{aligned}
& u_{t t x}(x, t)+u_{x}(x, t) \\
& =-\lim _{\delta \rightarrow 0}\left(\int_{0}^{x-\delta}+\int_{x-\delta}^{x+\delta}+\int_{x+\delta}^{1} K_{\xi x}(x, \xi)[\sigma(u \xi(\xi, t))\right. \\
& \left.\left.+\lambda u_{\xi t}(\xi, t)\right] \mathrm{d} \xi\right) \\
& =-\lim _{\delta \rightarrow 0}\left(\int_{0}^{x-\delta}+\int_{x+\delta}^{1} K_{\xi x}(x, \xi)\left[\sigma\left(u_{\xi}(\xi, t)\right)\right.\right. \\
& \left.\left.\quad-\sigma u_{\xi t}(\xi, t)\right] \mathrm{d} \xi\right)
\end{aligned}
$$

Multiplying both sides of (30) by $u_{x t}$ and using (6) of Lemma 4 we get

$$
\begin{aligned}
& \frac{1}{2} \frac{\mathrm{d}}{\mathrm{d} t}\left(u_{x t}^{2}+u_{x}^{2}+2 F\left(u_{x}\right)\right)+\lambda u_{x t}^{2} \\
& \leq C \int_{0}^{1}\left(\left|\sigma\left(u_{\xi}\right)\right|+\left|\lambda u_{\xi t}\right|\right) \mathrm{d} \xi\left|u_{x t}\right| .
\end{aligned}
$$

By condition (ii) of Theorem 2, the Young inequality, and inequality (28) we have

$$
\begin{aligned}
& \int_{0}^{1}\left(\left|\sigma\left(u_{\xi}\right)\right|+\left|\lambda u_{\xi_{t}}\right|\right) \mathrm{d} \xi \leq \\
& \int_{0}^{1}\left(C_{1} F\left(u_{\xi}\right)+C_{2}+\frac{1}{2}\left(u_{\xi_{t}}^{2}+\lambda^{2}\right)\right) \mathrm{d} \xi \leq C(T) .
\end{aligned}
$$

Substituting inequality (32) into inequality (31) and using the Young inequality we obtain

$$
\begin{aligned}
& \frac{\mathrm{d}}{\mathrm{d} t}\left(u_{x t}^{2}+u_{x}^{2}+2 F\left(u_{x}\right)\right) \\
& \leq C_{3}(T)+\left(2|\lambda|+\frac{1}{2}\right) u_{x t}^{2} .
\end{aligned}
$$

Here $C_{3}(T)$ is a constant dependent on $T$. Integrating (33) with respect to $t$ and using the Gronwall inequality we get

$$
\begin{aligned}
& \left\|u_{x t}\right\|_{\infty}^{2}+\left\|u_{x}\right\|_{\infty}^{2}+2\left\|F\left(u_{x}\right)\right\|_{\infty} \leq \\
& \left(\left\|u_{1}\right\|_{\infty}^{2}+\left\|u_{0 x}\right\|_{\infty}^{2}+2\left\|F\left(u_{0 x}\right)\right\|_{\infty}+C_{3}(T) T\right) \mathrm{e}^{\left(2|\lambda|+\frac{1}{2}\right) T} .
\end{aligned}
$$

By assumption (i) of Theorem 2 and the above inequality we obtain

$$
\left\|u_{x t}\right\|_{\infty}^{2}+\left\|u_{x}\right\|_{\infty}^{2} \leq C(T), \quad 0 \leq t \leq T .
$$

Using Lemma 3 and Lemma 1, it follows easily that

$$
\begin{aligned}
& \|\left(I-\frac{\partial^{2}}{\partial x^{2}}\right)^{-1}\left(\sigma\left(u_{x}\right)_{x}+\lambda u_{x x t}\right) \\
& \leq C\left(\|u\|_{2, p}+\left\|u_{t}\right\|_{2, p}\right) .
\end{aligned}
$$

From (9) and (35) we obtain

$$
\begin{aligned}
\|u\|_{2, p} \leq & \left\|u_{0}\right\|_{2, p}+\left\|u_{1}\right\|_{2, p} T \\
& +T \int_{0}^{t}\left[(C+1)\|u\|_{2, p}+C\left\|u_{\tau}\right\|_{2, p}\right] \mathrm{d} \tau, \\
\left\|u_{t}\right\|_{2, p} \leq & \left\|u_{1}\right\|_{2, p} \\
& +\int_{0}^{t}\left[(C+1)\|u\|_{2, p}+C\left\|u_{\tau}\right\|_{2, p}\right] \mathrm{d} \tau .
\end{aligned}
$$

Thus from (36) and (37) we have

$$
\begin{aligned}
\|u\|_{2, p}+\left\|u_{t}\right\|_{2, p} \leq & \left\|u_{0}\right\|_{2, p}+\left\|u_{1}\right\|_{2, p}(1+T) \\
& +C(1+T) \int_{0}^{t}\left(\|u\|_{2, p}+\left\|u_{\tau}\right\|_{2, p}\right) \mathrm{d} \tau .
\end{aligned}
$$

Applying the Gronwall inequality to the above inequality we obtain

$$
\|u\|_{2, p}+\left\|u_{t}\right\|_{2, p} \leq C(T), \quad 0 \leq t \leq T .
$$

From (25), (35), and (38) we have

$$
\begin{aligned}
& \left\|u_{t t}\right\|_{2, p}+\|u\|_{2, p} \leq C\left(\|u\|_{2, p}+\left\|u_{t}\right\|_{2, p}\right), \\
& \left\|u_{t t}\right\|_{2, p} \leq C(T), \quad 0 \leq t \leq T,
\end{aligned}
$$

and

$$
u(x, t) \in W^{2, \infty}\left([0, T] ; W^{2, p}[0,1] \cap W_{0}^{1, p}[0,1]\right) .
$$

By the arbitrariness of $T$ and (26), $T_{0}=\infty$, Theorem 2 is proved.

Theorem 3. If $\sigma^{\prime}(s)$ is bounded from below, i. e., there is a constant $C_{0}$ such that $\sigma^{\prime}(s) \geq C_{0}$ and $|\tilde{\sigma}(s)| \leq$ $C_{4} \int_{0}^{s} \tilde{\sigma}(y) \mathrm{d} y+C_{5}, s \in \mathrm{R}$ where $\tilde{\sigma}(s)=\sigma(s)-k_{0} s-$ $\sigma(0), k_{0}=\min \left\{C_{0}, 0\right\} \leq 0, C_{3}$ and $C_{4}$ are positive constants, then the conclusion of Theorem 2 also holds.

Proof. Let $\tilde{\sigma}(s)=\sigma(s)-k_{0} s-\sigma(0)$, where $k_{0}=$ $\min \left\{C_{0}, 0\right\} \leq 0$. Obviously $\tilde{\sigma}(0)=0, \tilde{\sigma}^{\prime}(s)=\sigma^{\prime}(s)-$ 
$k_{0} \geq 0$, and $\tilde{\sigma}(s)$ is a monotonically increasing function. Then $\tilde{F}(s)=\int_{0}^{s} \tilde{\sigma}(y) \mathrm{d} y \geq 0$. From (27) and noting that

$$
F(s)=\int_{0}^{s} \sigma(y) \mathrm{d} y=\int_{0}^{s}\left[\tilde{\sigma}(y)+k_{0} y+\sigma(0)\right] \mathrm{d} y,
$$

we have

$$
\begin{aligned}
& \|u(t)\|_{H^{1}}^{2}+\left\|u_{t}(t)\right\|_{H^{1}}^{2}+2 \int_{0}^{1} \tilde{F}\left(u_{x}\right) \mathrm{d} x \\
& \leq\left\|u_{0}\right\|_{H^{1}}^{2}+\left\|u_{1}\right\|_{H^{1}}^{2}+2 \int_{0}^{1} \tilde{F}\left(u_{0 x}\right) \mathrm{d} x \\
& \quad+k_{0}\left\|u_{0 x}\right\|_{2}^{2}+2|\lambda| \int_{0}^{t}\left\|u_{x \tau}(\tau)\right\|_{2}^{2} \mathrm{~d} \tau-k_{0}\left\|u_{x}(t)\right\|_{2}^{2} \\
& =\left\|u_{0}\right\|_{H^{1}}^{2}+\left\|u_{1}\right\|_{H^{1}}^{2}+2 \int_{0}^{1} \tilde{F}\left(u_{0 x}\right) \mathrm{d} x+k_{0}\left\|u_{0 x}\right\|_{2}^{2} \\
& \quad+2|\lambda| \int_{0}^{t}\left\|u_{x \tau}(\tau)\right\|_{2}^{2} \mathrm{~d} \tau-k_{0}\left\|u_{0}\right\|_{2}^{2}-2 k_{0} \int_{0}^{t}\left(u, u_{\tau}\right) \mathrm{d} \tau \\
& \leq\left\|u_{1}\right\|_{2}^{2}+\left(1-k_{0}\right)\left\|u_{0}\right\|_{2}^{2}+\left(1+k_{0}\right)\left\|u_{0 x}\right\|_{2}^{2}+\left\|u_{1 x}\right\|_{2}^{2} \\
& \quad+2 \int_{0}^{1} \tilde{F}\left(u_{0 x}\right) \mathrm{d} x \\
& \quad+\int_{0}^{t}\left[(2|\lambda|+1)\left\|u_{\tau}(\tau)\right\|_{2}^{2}+k_{0}^{2}\|u(\tau)\|_{2}^{2}\right] \mathrm{d} \tau .
\end{aligned}
$$

From the above inequality and the Gronwall inequality we get

$$
\begin{aligned}
& \|u(t)\|_{H^{1}}^{2}+\left\|u_{t}(t)\right\|_{H^{1}}^{2}+2 \int_{0}^{1} \tilde{F}\left(u_{x}\right) \mathrm{d} x \leq C(T), \\
& 0 \leq t \leq T .
\end{aligned}
$$

Therefore substituting $\sigma(s)=\tilde{\sigma}(s)+k_{0} s+\sigma(0)$ into (1) and the other, and repeating the proof in Theorem 2 leads to the conclusions of Theorem 3 .

Theorem 4. Assume that $u_{0}, u_{1} \in W^{k, p}[0,1] \cap$ $W_{0}^{1, p}[0,1], \sigma(s) \in C^{k}(\mathrm{R}), k>2,1<p<\infty$, and the following conditions hold:

(i) $\sigma(s) s \geq 0, \quad s \in \mathrm{R}$;

(ii) $|\sigma(s)| \leq C_{1} \int_{0}^{s} \sigma(y) \mathrm{d} y+C_{2}, \quad s \in \mathrm{R}$.

Then for any $T>0$ problem (1) -(3) admits a unique global solution $u(x, t) \in W^{2, \infty}\left([0, T] ; W^{k, p}[0,1] \cap\right.$ $\left.W_{0}^{1, p}[0,1]\right)$.

Proof. From Theorem 1 we know that problem (1)-(3) admits a unique local solution $u(x, t) \in$
$W^{2, \infty}\left(\left[0, T_{0}\right) ; W^{k, p}[0,1]\right.$. From the proof of the theorem we have $u(x, t) \in W^{2, \infty}\left([0, T] ; W^{2, p}[0,1] \cap W_{0}^{1, p}[0,1]\right)$, $\forall T>0$.

From (9) and (14), we have

$$
\begin{aligned}
\|u\|_{k, p} \leq & \left\|u_{0}\right\|_{k, p}+\left\|u_{1}\right\|_{k, p} T \\
& +T \int_{0}^{t}\left[(C+1)\|u\|_{k, p}+C\left\|u_{\tau}\right\|_{k, p}\right] \mathrm{d} \tau, \\
\left\|u_{t}\right\|_{k, p} \leq & \left\|u_{1}\right\|_{k, p} \\
& +\int_{0}^{t}\left[(C+1)\|u\|_{k, p}+C\left\|u_{\tau}\right\|_{k, p}\right] \mathrm{d} \tau .
\end{aligned}
$$

Adding inequalities (39) and (40), and applying the Gronwall inequality to the resulting inequality, we obtain

$$
\|u\|_{k, p}+\left\|u_{t}\right\|_{k, p} \leq C(T), \quad 0 \leq t \leq T .
$$

From (25), (14), and (41) we have

$$
\begin{aligned}
& \left\|u_{t t}\right\|_{k, p}+\|u\|_{k, p} \leq C\left(\|u\|_{k, p}+\left\|u_{t}\right\|_{k, p}\right), \\
& \left\|u_{t t}\right\|_{k, p} \leq C(T), \quad 0 \leq t \leq T,
\end{aligned}
$$

and

$$
u(x, t) \in W^{2, \infty}\left([0, T] ; W^{k, p}[0,1] \cap W_{0}^{1, p}[0,1]\right) .
$$

By the arbitrariness of $T$ and (26), $T_{0}=\infty$, Theorem 4 is proved.

\section{Asymptotic Behaviour of Solutions}

In this section, we discuss the asymptotic behaviour of the solutions for problem (1)-(3). For this purpose we define the energy by

$$
E(t)=\frac{1}{2}\left(\|u(t)\|_{H^{1}}^{2}+\left\|u_{t}(t)\right\|_{H^{1}}^{2}\right)+\int_{0}^{1} F\left(u_{x}\right) \mathrm{d} x,
$$

where $F(s)=\int_{0}^{s} \sigma(y) \mathrm{d} y$.

Theorem 5. Let $\lambda>0,1<p<\infty$ and assume that

(i) either $\sigma(s) s \geq 0$ or $\sigma^{\prime}(s) \geq C_{0}, s \in \mathrm{R}$, where $C_{0}$ is a constant;

(ii) $E(0)=\frac{1}{2}\left(\left\|u_{0}\right\|_{H^{1}}^{2}+\left\|u_{1}\right\|_{H^{1}}^{2}\right)+\int_{0}^{1} F\left(u_{0 x}\right) \mathrm{d} x>0$;

(iii) $D(s) \leq b \sigma(s) s, s \in \mathrm{R}$, where $b>0$ is a constant. 
Then for the global $W^{2, p}$ solution $u(x, t)$ of problem (1) - (3) there exist $\delta_{1}>0$ and $M>0$ such that

$$
\|u(t)\|_{H^{1}}^{2}+\left\|u_{t}(t)\right\|_{H^{1}}^{2}+2 \int_{0}^{1} F\left(u_{x}\right) \mathrm{d} x \leq M E(0) \mathrm{e}^{-\delta_{1} t},
$$$$
t>0 \text {. }
$$

Proof. Let $u(x, t)$ be a global $W^{2, p}$ solution of problem (1) - (3). Taking the $L^{2}$ inner product of (1) with $u_{t}$ it follows that

$$
\frac{\mathrm{d}}{\mathrm{d} t} E(t)+\lambda\left\|u_{x t}(t)\right\|_{2}^{2}=0, \quad t>0 .
$$

Multiplying (44) by $\mathrm{e}^{\delta t}$ gives

$$
\frac{\mathrm{d}}{\mathrm{d} t}\left(\mathrm{e}^{\delta t} E(t)\right)+\lambda \mathrm{e}^{\delta t}\left\|u_{x t}(t)\right\|_{2}^{2}=\delta \mathrm{e}^{\delta t} E(t), \quad t>0 .
$$

Integrating (45) over $(0, t)$ we get

$$
\begin{aligned}
& \mathrm{e}^{\delta t} E(t)+\lambda \int_{0}^{t} \mathrm{e}^{\delta \tau}\left\|u_{x \tau}(\tau)\right\|_{2}^{2} \mathrm{~d} \tau= \\
& E(0)+\frac{\delta}{2} \int_{0}^{t} \mathrm{e}^{\delta \tau}\left(\left\|u_{\tau}(\tau)\right\|_{2}^{2}+\left\|u_{x \tau}(\tau)\right\|_{2}^{2}\right) \mathrm{d} \tau \\
& +\delta \int_{0}^{t} \mathrm{e}^{\delta \tau}\left(\frac{1}{2}\|u(\tau)\|_{2}^{2}+\frac{1}{2}\left\|u_{x}(\tau)\right\|_{2}^{2}+\int_{0}^{1} F\left(u_{x}\right) \mathrm{d} x\right) \mathrm{d} \tau,
\end{aligned}
$$$$
t>0 \text {. }
$$

Case 1. If $\sigma(s) s \geq 0, s \in \mathrm{R}$, then $F(s) \geq 0$. Thus from assumption (iii) of Theorem 5 we have $0 \leq$ $F(s) \leq b \sigma(s) s$. Using this relation and (1) we obtain

$$
\begin{aligned}
& \int_{0}^{t} \mathrm{e}^{\delta \tau}\left(\frac{1}{2}\|u(\tau)\|_{2}^{2}+\frac{1}{2}\left\|u_{x}(\tau)\right\|_{2}^{2}+\int_{0}^{1} F\left(u_{x}\right) \mathrm{d} x\right) \mathrm{d} \tau \\
& \leq b_{1} \int_{0}^{t} \mathrm{e}^{\delta \tau}\left(\|u(\tau)\|_{2}^{2}+\left\|u_{x}(\tau)\right\|_{2}^{2}+\int_{0}^{1} \sigma\left(u_{x}\right) u_{x} \mathrm{~d} x\right) \mathrm{d} \tau \\
& =-b_{1} \int_{0}^{t} \mathrm{e}^{\delta \tau}\left[\left(u_{\tau \tau}, u\right)+\left(u_{x \tau \tau}, u_{x}\right)+\frac{\lambda}{2} \frac{\mathrm{d}}{\mathrm{d} \tau}\left\|u_{x}(\tau)\right\|_{2}^{2}\right] \mathrm{d} \tau \\
& =-b_{1}\left[\mathrm{e}^{\delta \tau}\left(\left(u_{t}, u\right)+\left(u_{x t}, u_{x}\right)+\frac{\lambda}{2}\left\|u_{x}(t)\right\|_{2}^{2}\right)\right. \\
& -\left(\left(u_{1}, u_{0}\right)+\left(u_{1 x}, u_{0 x}\right)+\frac{\lambda}{2}\left\|u_{0 x}\right\|_{2}^{2}\right) \\
& -\int_{0}^{t} \mathrm{e}^{\delta \tau}\left(\left\|u_{\tau}(\tau)\right\|_{2}^{2}+\left\|u_{x \tau}(\tau)\right\|_{2}^{2}\right) \mathrm{d} \tau \\
& \left.+\delta \int_{0}^{t} \mathrm{e}^{\delta \tau}\left(\left(u_{\tau}, u\right)+\left(u_{x \tau}, u_{x}\right)+\frac{\lambda}{2}\left\|u_{x}(\tau)\right\|_{2}^{2}\right) \mathrm{d} \tau\right] \\
& \leq 2 b_{1} \int_{0}^{t} \mathrm{e}^{\delta \tau}\left\|u_{x \tau}(\tau)\right\|_{2}^{2} \mathrm{~d} \tau+(1+\lambda) b_{1} \mathrm{e}^{\delta t} E(t) \\
& +(1+\lambda) b_{1} E(0)+(1+\lambda) b_{1} \delta \int_{0}^{t} \mathrm{e}^{\delta \tau} E(\tau) \mathrm{d} \tau, \\
& t>0 \text {, }
\end{aligned}
$$

where $b_{1}=\max \left\{\frac{1}{2}, b\right\}$. Substituting inequality (47) into (46) we obtain

$$
\begin{aligned}
& \mathrm{e}^{\delta t} E(t)+\lambda \int_{0}^{t} \mathrm{e}^{\delta \tau}\left\|u_{x \tau}(t)\right\|_{2}^{2} \mathrm{~d} \tau \leq \\
& \left(1+(1+\lambda) b_{1} \delta\right) E(0)+\left(1+2 b_{1}\right) \delta \int_{0}^{t} \mathrm{e}^{\delta \tau}\left\|u_{x \tau}(t)\right\|_{2}^{2} \mathrm{~d} \tau \\
& +(1+\lambda) b_{1} \delta \mathrm{e}^{\delta t} E(t)+(1+\lambda) b_{1} \delta^{2} \int_{0}^{t} \mathrm{e}^{\delta \tau} E(\tau) \mathrm{d} \tau \\
& t>0 .
\end{aligned}
$$

Take $\delta: 0<\delta<\min \left\{\frac{\lambda}{1+2 b_{1}}, \frac{1}{(1+\lambda) b_{1}}\right\}$, we deduce from (48) that

$$
\mathrm{e}^{\delta t} E(t) \leq \frac{M}{2} E(0)+\theta \delta \int_{0}^{t} \mathrm{e}^{\delta \tau} E(\tau) \mathrm{d} \tau,
$$

where $\frac{M}{2}=\frac{1+(1+\lambda) b_{1} \delta}{1-(1+\lambda) b_{1} \delta}$ and $\theta=\frac{(1+\lambda) b_{1} \delta}{1-(1+\lambda) b_{1} \delta}<1$. Applying the Gronwall inequality to (49) we obtain the result of (43) for $\delta_{1}=(1-\theta) \delta>0$.

Case 2. If $\sigma^{\prime}(s) \geq C_{0}, s \in \mathrm{R}$, let $\tilde{\sigma}(s)=\sigma(s)-k_{0} s-$ $\sigma(0)$, where $k_{0}=\min \left\{C_{0}, 0\right\} \leq 0$. Obviously $\tilde{\sigma}(0)=0$, $\tilde{\sigma}^{\prime}(s)=\sigma^{\prime}(s)-k_{0} \geq 0, \tilde{\sigma}(s) s \geq 0, s \in \mathrm{R}$, and if assumption (iii) of Theorem 5 holds, then a simple calculation shows that $0 \leq \tilde{F}(s)=\int_{0}^{s} \tilde{\sigma}(y) \mathrm{d} y \leq b \tilde{\sigma}(s) s$, $s \in \mathrm{R}$. Therefore substituting $\sigma(s)=\tilde{\sigma}(s)+k_{0} s+\sigma(0)$ into (1) and repeating the proof of Case 1 implies the conclusions of Theorem 5. The theorem thus is proved.

\section{Blow up of Solutions}

In this section, we consider the blow up of solutions for problem (1)-(3). For this purpose, we define the energy by (42).

Theorem 6. Assume that

(i) $\sigma(s) \in C^{k}(\mathrm{R}), \sigma(s) s \leq \alpha F(s) \leq-\alpha \beta|s|^{m+1}, k \geq$ $2, s \in \mathrm{R}$, where $\alpha>2, \beta>0$ and $m>1$ are constants;

(ii) $u_{0}, u_{1} \in W^{k, p}[0,1] \cap W_{0}^{1, p}[0,1], 1<p<\infty$ such that the initial energy

$$
\text { (iii) } \begin{aligned}
E(0)= & \frac{1}{2}\left(\left\|u_{0}\right\|_{H^{1}}^{2}+\left\|u_{1}\right\|_{H^{1}}^{2}\right) \\
& +\int_{0}^{1} F\left(u_{0 x}\right) \mathrm{d} x<0 .
\end{aligned}
$$

Then the $W^{k, p}$ solution $u(x, t)$ blows up in finite time $\tilde{T}$, that is

$$
\begin{aligned}
& \|u(t)\|_{H^{1}}^{2}+\left\|u_{t}(t)\right\|_{H^{1}}^{2}+\lambda \int_{0}^{t}\left\|u_{x}(\tau)\right\|_{2}^{2} \mathrm{~d} \tau \rightarrow \infty \\
& \text { as } t \rightarrow \tilde{T}^{-}
\end{aligned}
$$


where $\tilde{T}$ is different for different conditions with obtain

$\lambda \geq 0$.

Proof. By multiplying (1) by $u_{t}$ and integrating the new equation in the interval $(0,1)$ we obtain

$$
E^{\prime}(t)+\lambda\left\|u_{x t}(t)\right\|_{2}^{2}=0, E(t) \leq E(0)<0, t \geq 0 .
$$

Let

$$
H(t)=\|u(t)\|_{2}^{2}+\left\|u_{x}(t)\right\|_{2}^{2}+\lambda \int_{0}^{t}\left\|u_{x}(\tau)\right\|_{2}^{2} \mathrm{~d} \tau
$$

then

$$
\begin{gathered}
H^{\prime}(t)=2\left(u, u_{t}\right)+2\left(u_{x}, u_{x t}\right)+\lambda\left\|u_{x}(t)\right\|_{2}^{2}, \quad(54) \\
H^{\prime \prime}(t)=2\left(\left\|u_{t}(t)\right\|_{2}^{2}+\left\|u_{x t}(t)\right\|_{2}^{2}+\int_{0}^{1} u_{x} u_{x t t} \mathrm{~d} x\right) \\
+2 \int_{0}^{1} u\left(u_{x x}+u_{x x t}+\lambda u_{x x t}-u+\left(\sigma\left(u_{x}\right)\right)_{x}\right) \mathrm{d} x \\
+\frac{\mathrm{d}}{\mathrm{d} t} \lambda\left\|u_{x}(t)\right\|_{2}^{2} \\
=2\left(\left\|u_{t}(t)\right\|_{2}^{2}+\left\|u_{x t}(t)\right\|_{2}^{2}-\left\|u_{x}(t)\right\|_{2}^{2}-\|u(t)\|_{2}^{2}\right. \\
\left.\quad-\int_{0}^{1} u_{x} \sigma\left(u_{x}\right) \mathrm{d} x\right) \\
\geq 2\left(\left\|u_{t}(t)\right\|_{2}^{2}+\left\|u_{x t}(t)\right\|_{2}^{2}-\left\|u_{x}(t)\right\|_{2}^{2}-\|u(t)\|_{2}^{2}\right. \\
\left.-\alpha \int_{0}^{1} F\left(u_{x}\right) \mathrm{d} x\right) \\
\geq 2\left(2\left\|u_{t}(t)\right\|_{2}^{2}+2\left\|u_{x t}(t)\right\|_{2}^{2}-(\alpha-2) \int_{0}^{1} F\left(u_{x}\right) \mathrm{d} x\right. \\
-2 E(0)) \\
\geq 2\left(2\left\|u_{t}(t)\right\|_{2}^{2}+2\left\|u_{x t}(t)\right\|_{2}^{2}+(\alpha-2) \beta\left\|u_{x}(t)\right\|_{m+1}^{m+1}\right. \\
-2 E(0)), \quad t>0,
\end{gathered}
$$

$$
\begin{aligned}
& H^{\prime}(t) \geq \\
& 2(\alpha-2) \beta \int_{0}^{t}\left\|u_{x}(\tau)\right\|_{m+1}^{m+1} \mathrm{~d} \tau-4 E(0) t+H^{\prime}(0), \\
& t>0
\end{aligned}
$$

After this calculation, we could add (55) with (56). Then we get

$$
\begin{aligned}
& H^{\prime \prime}(t)+H^{\prime}(t) \\
& \geq 4\left\|u_{t}(t)\right\|_{2}^{2}+4\left\|u_{x t}(t)\right\|_{2}^{2} \\
& \quad+2(\alpha-2) \beta\left(\left\|u_{x}(t)\right\|_{m+1}^{m+1}+\int_{0}^{t}\left\|u_{x}(\tau)\right\|_{m+1}^{m+1} \mathrm{~d} \tau\right) \\
& \quad-4 E(0)(1+t)+H^{\prime}(0)=g(t), \quad t>0 .
\end{aligned}
$$

Take $r=\frac{m+3}{2}$, obviously $2<r<m+1$ and $r^{\prime}=\frac{m+3}{m+1}$ $(<2)$. By using the Young inequality and the SobolevPoincaré inequality, we get

$$
\begin{aligned}
&\left|\left(u, u_{t}\right)\right| \leq \frac{1}{r}\|u(t)\|_{r}^{r}+\frac{1}{r^{\prime}}\left\|u_{t}(t)\right\|_{r^{\prime}}^{r^{\prime}} \\
& \leq C_{1}\left[\left(\left\|u_{x}(t)\right\|_{m+1}^{m+1}\right)^{\mu}+\left(\left\|u_{t}(t)\right\|_{2}^{2}\right)^{\mu}\right], \\
&\left|\left(u, u_{t}\right)\right|^{\frac{1}{\mu}} \leq C_{2}\left[\left\|u_{x}(t)\right\|_{m+1}^{m+1}+\left\|u_{t}(t)\right\|_{2}^{2}\right], \quad t>0,
\end{aligned}
$$

and similarly

$$
\mid\left(u_{x}, u_{x t}\right)^{\frac{1}{\mu}} \leq C_{3}\left[\left\|u_{x}(t)\right\|_{m+1}^{m+1}+\left\|u_{x t}(t)\right\|_{2}^{2}\right], t>0,
$$

where in this inequality and in the sequel $C_{i}(i=$ $1,2, \ldots)$ denote positive constants independent of $t$, $\mu=\frac{m+3}{2(m+1)}(<1)$. By the Sobolev-Poincaré inequality and the Hölder inequality

$$
\begin{aligned}
\left\|u_{x}(t)\right\|_{m+1}^{m+1} & \geq\left(\|u(t)\|_{2}^{2}\right)^{\frac{m+1}{2}}, \quad t>0, \\
\left\|u_{x}(t)\right\|_{m+1}^{m+1} & \geq\left(\left\|u_{x}(t)\right\|_{2}^{2}\right)^{\frac{m+1}{2}}, \quad t>0, \\
\int_{0}^{t}\left\|u_{x}(\tau)\right\|_{m+1}^{m+1} \mathrm{~d} \tau & \geq t^{\frac{1-m}{2}}\left(\int_{0}^{t}\left\|u_{x}(\tau)\right\|_{2}^{2} \mathrm{~d} \tau\right)^{\frac{m+1}{2}},
\end{aligned}
$$

where the assumption (i) of Theorem 6 and the fact that

$$
\begin{aligned}
& \alpha \int_{0}^{1} F\left(u_{x}\right) \mathrm{d} x \leq 2 E(0)-\left\|u_{t}(t)\right\|_{2}^{2}-\left\|u_{x}(t)\right\|_{2}^{2} \\
& -\left\|u_{x t}(t)\right\|_{2}^{2}-\|u(t)\|_{2}^{2}+(\alpha-2) \int_{0}^{1} F\left(u_{x}\right) \mathrm{d} x
\end{aligned}
$$

have been used. Taking (55) and integrating this, we $t>0$.

(1) If $\lambda>0$, by using the inequalities (58)-(62), we obtain

$$
\begin{aligned}
g(t) \geq & C_{4}\left(4\left\|u_{x}(t)\right\|_{m+1}^{m+1}+\left\|u_{t}(t)\right\|_{2}^{2}+\left\|u_{x t}(t)\right\|_{2}^{2}\right. \\
& \left.+\int_{0}^{t}\left\|u_{x}(\tau)\right\|_{m+1}^{m+1} \mathrm{~d} \tau\right)-4 E(0) t+H^{\prime}(0)
\end{aligned}
$$




$$
\begin{aligned}
\geq & C_{5}\left(\left|\left(u, u_{t}\right)\right|^{\frac{1}{\mu}}+\left|\left(u_{x}, u_{x t}\right)\right|^{\frac{1}{\mu}}+\left(\|u(t)\|_{2}^{2}\right)^{\frac{m+1}{2}}\right. \\
& \left.+\left(\left\|u_{x}(t)\right\|_{2}^{2}\right)^{\frac{m+1}{2}}+t^{\frac{1-m}{2}}\left(\int_{0}^{t}\left\|u_{x}(\tau)\right\|_{2}^{2} \mathrm{~d} \tau\right)^{\frac{m+1}{2}}\right) \\
& -4 E(0) t+H^{\prime}(0) \\
\geq & C_{6} t^{\frac{1-m}{2}}\left(\left|\left(u, u_{t}\right)\right|^{\gamma}+\left|\left(u_{x}, u_{x t}\right)\right|^{\gamma}+\left(\|u(t)\|_{2}^{2}\right)^{\gamma}\right. \\
& \left.+\left(\left\|u_{x}(t)\right\|_{2}^{2}\right)^{\gamma}+\left(\int_{0}^{t}\left\|u_{x}(\tau)\right\|_{2}^{2} \mathrm{~d} \tau\right)^{\gamma}\right) \\
& -4 E(0) t+H^{\prime}(0)-C_{6} t^{\frac{1-m}{2}}, \quad t \geq 1,
\end{aligned}
$$

where in this inequality and in the sequel $\gamma=\frac{1}{\mu}>1$. Since $-4 E(0) t+H^{\prime}(0)-C_{6} t^{\frac{1-m}{2}} \rightarrow \infty$ as $t \rightarrow \infty$, there must be a $t_{1} \geq 1$ such that

$$
-4 E(0) t+H^{\prime}(0)-C_{6} t^{\frac{1-m}{2}} \geq 0 \text { as } t \geq t_{1} .
$$

Let

$$
y(t)=H^{\prime}(t)+H(t),
$$

then from (56) and (53) we obtain $y(t)>0$ as $t \geq t_{1}$. By using the inequality

$$
\left(a_{1}+\ldots+a_{l}\right)^{n} \leq 2^{(n-1)(l-1)}\left(a_{1}^{n}+\ldots+a_{l}^{n}\right),
$$

where $a_{i} \geq 0(i=1, \ldots, l)$ and $n>1$ are real numbers, the fact (64) and using (63), we get

$$
g(t) \geq C_{6} t^{\frac{1-m}{2}} y^{\gamma}(t), \quad t \geq t_{1} .
$$

So combining (57) with (66) gives

$$
y^{\prime}(t) \geq C_{6} t^{\frac{1-m}{2}} y^{\gamma}(t), \quad t \geq t_{1} .
$$

Therefore, there exists a positive constant

$$
\tilde{T} \leq\left[\begin{array}{ll}
{\left[t_{1}^{\frac{3-m}{2}}+\frac{3-m}{2 C_{6}(\gamma-1) y^{\gamma-1}\left(t_{1}\right)}\right]^{\frac{2}{3-m}},} & m \neq 3, \\
t_{1} \cdot \exp \frac{1}{C_{6}(\gamma-1) y^{\gamma-1}\left(t_{1}\right)}, & m=3,
\end{array}\right.
$$

such that

$$
y(t) \rightarrow \infty \text { as } t \rightarrow \tilde{T}^{-} .
$$

By using (53), (54), and (69), we find

$2\|u(t)\|_{2}^{2}+\left\|u_{t}(t)\right\|_{2}^{2}+(\lambda+2)\left\|u_{x}(t)\right\|_{2}^{2}+\left\|u_{x t}(t)\right\|_{2}^{2}$

$+\lambda \int_{0}^{t}\left\|u_{x}(\tau)\right\|_{2}^{2} \mathrm{~d} \tau \geq H^{\prime}(t)+H(t) \rightarrow \infty$
So (70) implies (51).

(2) If $\lambda=0$ by using the inequalities (58)-(61), we obtain

$$
\begin{aligned}
g(t) \geq & C_{7}\left(4\left\|u_{x}(t)\right\|_{m+1}^{m+1}+\left\|u_{t}(t)\right\|_{2}^{2}+\left\|u_{x t}(t)\right\|_{2}^{2}+1\right) \\
& -4 E(0) t+H^{\prime}(0) \\
\geq & C_{8}\left[\left|\left(u, u_{t}\right)\right|^{\gamma}+\left|\left(u_{x}, u_{x t}\right)\right|^{\gamma}+\left(\|u(t)\|_{2}^{2}\right)^{\gamma}\right. \\
& \left.+\left(\left\|u_{x}(t)\right\|_{2}^{2}\right)^{\gamma}\right]-4 E(0) t+H^{\prime}(0) \\
t>0 &
\end{aligned}
$$

By the same method as used in deriving (67), there must be a $t_{2}>0$ such that $-4 E(0) t+H^{\prime}(0)>0$ and $y(t)=H^{\prime}(t)+H(t)>0$ as $t \geq t_{2}$. So combining (57) with (71) yields

$$
y^{\prime}(t) \geq C_{8} y^{\gamma}(t), \quad t \geq t_{2} .
$$

Equation (72) implies that there exists a positive constant $\tilde{T}=t_{2}+\left[C_{9}(\gamma-1) y^{\gamma-1}\left(t_{2}\right)\right]^{-1}$ such that $y(t) \rightarrow$ $\infty$ as $t \rightarrow \tilde{T}^{-}$. Since $y(t) \leq 2\|u(t)\|_{2}^{2}+\left\|u_{t}(t)\right\|_{2}^{2}+$ $2\left\|u_{x}(t)\right\|_{2}^{2}+\left\|u_{x t}(t)\right\|_{2}^{2},(51)$ is satisfied. This completes the proof.

Theorem 7. Suppose that the conditions (i) and (ii) of Theorem 6 hold and one of the following conditions are valid:

(i) $E(0)=0$, and, if $\lambda>0, H^{\prime}(0) \geq C_{10} t^{\frac{1-m}{2}}$ for $t \geq$ 1 , and, if $\lambda=0, H^{\prime}(0)>0$ for $t>0$.

(ii) $E(0)>0$, and, if $\lambda>0, H^{\prime}(0) \geq 4 E(0)(1+t)+$ $C_{10} t^{\frac{1-m}{2}}$ for some $t_{3} \geq 1$, and, if $\lambda=0, H^{\prime}(0)>$ $4 E(0)(1+t)$ for some $t_{4}>0$.

Then the $W^{k, p}$ solution $u(x, t)$ blows up in finite time $\tilde{T}$.

Proof. At first we assume that condition (i) holds.

(1) If $\lambda>0$ then using inequalities (58)-(62), we obtain

$$
\begin{aligned}
g(t) \geq & C_{9} t^{\frac{1-m}{2}}\left(\left|\left(u, u_{t}\right)\right|^{\gamma}+\left|\left(u_{x}, u_{x t}\right)\right|^{\gamma}+\left(\|u(t)\|_{2}^{2}\right)^{\gamma}\right. \\
& \left.+\left(\left\|u_{x}(t)\right\|_{2}^{2}\right)^{\gamma}+\left(\int_{0}^{t}\left\|u_{x}(\tau)\right\|_{2}^{2} \mathrm{~d} \tau\right)^{\gamma}\right) \\
& -4 E(0)(1+t)+H^{\prime}(0)-C_{9} t^{\frac{1-m}{2}}, \quad t \geq 1 .
\end{aligned}
$$

From condition (i) we have

$$
\begin{aligned}
& -4 E(0)(1+t)+H^{\prime}(0)-C_{9} t^{\frac{1-m}{2}}=H^{\prime}(0)-C_{9} t^{\frac{1-m}{2}} \\
& \geq 0 \text { as } t \geq 1 .
\end{aligned}
$$


Thus, we obtain $y(t)>0$ as $t \geq 1$. By the similar method as used in deriving (67), we find

$$
y^{\prime}(t) \geq C_{10} t^{\frac{1-m}{2}} y^{\gamma}(t), \quad t \geq 1 .
$$

Therefore there exists a positive constant

$$
\tilde{T} \leq\left[\begin{array}{ll}
{\left[1+\frac{3-m}{2 C_{9}(\gamma-1) y^{\gamma-1}(1)}\right]^{\frac{2}{3-m}},} & m \neq 3, \\
\exp \frac{1}{C_{9}(\gamma-1) y^{\gamma-1}(1)}, & m=3,
\end{array}\right.
$$

such that $y(t) \rightarrow \infty$ as $t \rightarrow \tilde{T}^{-}$.

(2) If $\lambda=0$ then using inequalities (58)-(61), we obtain

$$
\begin{aligned}
g(t) \geq & C_{10}\left[\left|\left(u, u_{t}\right)\right|^{\gamma}+\left|\left(u_{x}, u_{x t}\right)\right|^{\gamma}+\left(\|u(t)\|_{2}^{2}\right)^{\gamma}\right. \\
& \left.+\left(\left\|u_{x}(t)\right\|_{2}^{2}\right)^{\gamma}\right]-4 E(0)(1+t)+H^{\prime}(0), \\
t>0 . &
\end{aligned}
$$

From condition (i), we have

$$
-4 E(0)(1+t)+H^{\prime}(0)=H^{\prime}(0)>0 \text { as } t>0 .
$$

Thus we obtain $y(t)>0$ as $t>0$. As a result, we get

$$
y^{\prime}(t) \geq C_{11} y^{\gamma}(t), \quad t>0 .
$$

Equation (79) implies that there exists a positive constant $\tilde{T} \leq\left[C_{11}(\gamma-1) y^{\gamma-1}(0)\right]^{-1}$ such that $y(t) \rightarrow \infty$ as $t \rightarrow \tilde{T}^{-}$.

[1] Z. Weiqiu, Acta Solid Mech. Sinica 1, 247 (1980).

[2] G. Andrews, J. Diff. Eq. 35, 200 (1980).

[3] D. D. Ang and A. P.N. Dinh, SIAM J. Math. Anal. 19, 1409 (1988).

[4] M. Can, S. R. Park, and F. Aliyev, J. Math. Anal. Appl. 213, 540 (1997).

[5] P. Constantin and J.C. Saut, J. Am. Math. Soc. 1, 1413 (1988).

[6] C. M. Dafermos, J. Diff. Eq. 6, 71 (1969).

[7] D. Erdem, Appl. Math. Lett. 12, 65 (1999).

[8] D. Erdem and V. K. Kalantarov, Appl. Math. Lett. 15, 585 (2002).

[9] J. M. Greenberg, J. Math. Anal. Appl. 25, 575 (1969).

[10] J. M. Greenberg and R.C. Maccamy, J. Math. Anal. Appl. 31, 406 (1970).

[11] S. Kawashima and Y. Shibata, Comm. Math. Phys. 148, 189 (1992).

[12] S. Lai and Y. Wu, Discrete Cont. Dyn. S. B 3, 401 (2003).
Second, we assume that condition (ii) holds.

(1) If $\lambda>0$ from condition (ii) we have

$$
-4 E(0)(1+t)+H^{\prime}(0)-C_{10} t^{\frac{1-m}{2}} \geq 0 \text { as } t \geq t_{3} .
$$

By use of inequalities (58)-(62) and (80), we obtain $y(t)>0$ as $t>t_{3}$. Thus, we find

$$
y^{\prime}(t) \geq C_{10} t^{\frac{1-m}{2}} y^{\gamma}(t), \quad t \geq t_{3} .
$$

Therefore, there exists a positive constant

$$
\tilde{T} \leq\left[\begin{array}{ll}
{\left[t_{3}^{\frac{3-m}{2}}+\frac{3-m}{2 C_{10}(\gamma-1) y^{\gamma-1}\left(t_{3}\right)}\right]^{\frac{2}{3-m}},} & m \neq 3, \\
t_{3} \cdot \exp \frac{1}{C_{10}(\gamma-1) y^{\gamma-1}\left(t_{3}\right)}, & m=3,
\end{array}\right.
$$

such that $y(t) \rightarrow \infty$ as $t \rightarrow \tilde{T}^{-}$.

(2) If $\lambda=0$ from condition (ii) we have

$$
-4 E(0)(1+t)+H^{\prime}(0)>0 \text { as } t \geq t_{4} .
$$

By use of inequalities (58)-(61) and (83), we obtain $y(t)>0$ as $t \geq t_{4}$. It follows that

$$
y^{\prime}(t) \geq C_{11} y^{\gamma}(t), \quad t \geq t_{4} .
$$

Equation (84) implies that there exists a positive constant $\tilde{T} \leq t_{4}+\left[C_{11}(\gamma-1) y^{\gamma-1}\left(t_{4}\right)\right]^{-1}$ such that $y(t) \rightarrow$ $\infty$ as $t \rightarrow \tilde{T}^{-}$. The theorem is proved.

[13] S. Lai, Y. Wu, and X. Yang, Commun. Pure Appl. Anal. 3, 319 (2004)

[14] N. T. Lang and A. P. N. Dinh, Nonlinear Anal. 19, 613 (1992).

[15] S. P. Levandosky, J. Diff. Eq. 143, 360 (1998).

[16] Y. A. Li and P. J. Olver, J. Diff. Eq. 162, 27 (2000).

[17] H. A. Levine, Trans. Am. Math. Soc. 192, 1 (1974).

[18] H. A. Levine and J. Serrin, Arch. Rational Mech. Anal. 137, 341 (1997).

[19] M. Nakao and K. Ono, Math. Z. 214, 325 (1993).

[20] K. Nishihara, J. Diff. Eq. 137, 384 (1997).

[21] K. Ono, Math. Meth. Appl. Sci. 23, 535 (2000).

[22] H. Pecher, Math. Z. 185, 245 (1984).

[23] N. Polat, D. Kaya, and H. İ. Tutalar, in: Dynamical Systems and Applications, GBS Publishers and Distributors, India 2005, p. 572.

[24] N. Polat, D. Kaya, and H. I. Tutalar, Z. Naturforsch. 60a, 473 (2005).

[25] N. Polat and D. Kaya, Z. Naturforsch. 61a, 235 (2006). 
[26] N. Polat, Z. Naturforsch. 63a, 543 (2008).

[27] P. Rybka and K. H. Hoffman, J. Math. Anal. Appl. 226, 61 (1998).

[28] V. V. Varlamov, Int. J. Maths. Math. Sci. 22, 131 (1999).

[29] V. V. Varlamov, Discrete Cont. Dyn. S. 7, 675 (2001).

[30] S. Wang and G. Chen, Nonlinear Anal. 64, 159 (2006).

[31] L. Yacheng and Z. Junsheng, Nonlinear Anal. 62, 245 (2005).

[32] Y. Zhijian and S. Changming, Nonlinear Anal. The. Meth. Appl. 28, 2017 (1997).

[33] Y. Zhijian, J. Diff. Eq. 187, 520 (2003).

[34] Y. Zhijian, J. Math. Anal. Appl. 313, 197 (2006).

[35] Z. Shanyuan and Z. Wei, Acta Mech. Sinica 20, 58 (1988).
[36] Z. Wei and Y. Guitong, Appl. Math. Mech. 7, 571 (1986).

[37] C. Guowang and W. Shubin, Comment. Math. Univ. Carolinae 36, 475 (1995).

[38] Y.-L. Zhou and H.-Y. Fu, Acta Math. Sinica 26, 234 (1983).

[39] I. Kocev, Math. Sb. 38, 360 (1956).

[40] J.F. Rodrigues, Obstacle Problems in Mathematical Physics, North-Holland, Amsterdam, New York 1987.

[41] C. Guowang and W. Shubin, Nonlinear Anal. 36, 961 (1999).

[42] M. A. Naemark, Linear Differential Operators, Publishing House of Technology and Theory, Gaustyhisdute 1954 (in Russian).

[43] E. Zauderer, Partial Differential Equations of Applied Mathematics, Wiley, New York 1983. 